\title{
«Una beffa [...] fatta da una donna a uno solenne religioso»: l'arte della manipolazione verbale ed emotiva nel Decameron III 3
}

\author{
"Una beffa [...] fatta da una donna a uno solenne religioso": \\ the Art of Verbal and Emotional Manipulation \\ in The Decameron III 3
}

\author{
Maria Maślanka-Soro \\ Uniwersytet Jagielloński \\ maria.maslanka-soro@uj.edu.pl
}

\begin{abstract}
Boccaccio's Decameron is, as we know, the supreme achievement of the medieval narratio brevis. The art of speaking and, in general, of the proficient use of words, is what distinguishes the "gentile brigata" of ten young men and women who articulate and advance the new ars narrandi. Its role is crucial in many of the novellas in The Decameron: thanks to their capacity to persuade, or rather to manipulate their listeners verbally, many of its male and female protagonists manage to avoid dangers and unpleasant situations, or to get what they want: love, money, or other advantages. Particularly two of the male protagonists, ser Ciappelletto and frate Cipolla, excel others in verbal fraud and mockery.

The anonymous protagonist of the third novella of the Third Day is neither as famous nor as examined by critics, even though she is very clever in her rhetorical and theatrical skills. The purpose of this essay is to analyse the strategies of verbal and emotional manipulation which lead her to seduce a "valoroso uomo di mezza età" with the involuntary help of the simple-minded and credulous friar.
\end{abstract}

Keywords: Boccaccio, The Decameron, verbal and emotional manipulation, theatricality, mockery (beffa)

Il Decameron è, com'è risaputo, una raccolta di cento novelle (esattamente centouno con quella raccontata dall'autore nell'Introduzione alla Quarta Giornata) che grazie alla cornice ${ }^{1}$ - la narrazione principale ovvero la storia portante che ha come

\footnotetext{
${ }^{1}$ Sulla cornice nel capolavoro del Boccaccio e in particolare sui suoi tre tipi, cf. Picone, 2004a, pp. 9-31; lì si veda un'ulteriore bibliografia.
} 
protagonisti sette donne e tre uomini di alta borghesia fiorentina, diventati a loro volta narratori di novelle - riceve una struttura continua e finita. Questa tecnica (di origine orientale, presente ad esempio nel Libro di Sindbad o dei sette savi, largamente conosciuto nel medioevo europeo) permette di fondere insieme i racconti caratterizzati da una varietas riguardante il rapporto con la realtà (da storico o metastorico a leggendario e favoloso), la tematica (oscillante tra il quotidiano e l'avventuroso o anche il meraviglioso), l'ambiente (prevalentemente borghese, ma anche di corte, più raramente popolano, a volte quello «misto»), il tono (dal comico al tragico), lo stile (dall'alto al basso, con una certa predilezione per il medioalto).

La funzione delle rubriche autoriali è quella di consentire la classificazione dei microtesti novellistici all'interno del macrotesto decameroniano - classificazione che si fonda sull'elemento tematico dominante in ciascuna delle decadi a eccezione della prima e penultima, anche se nel caso della prima il problema non è così semplice. Infatti, in alternativa a queste esplicite indicazioni che introducono un determinato ordine tra le novelle di ogni giornata, alcuni studiosi hanno individuato altre possibili divisioni in base a criteri anche formali e strutturali, tra le quali la suddivisione in due parti (Stewart, 1986, pp. 19-38; Picone, 1993, pp. 633-637), introdotte rispettivamente dalla prima novella della Prima Giornata e dalla prima novella della Sesta Giornata. Nel fare ciò, viene preso in considerazione l'interesse dell'autore verso il potere della parola, intorno al quale sono costruite le vicende narrate in entrambe le decadi, e inoltre il carattere metaletterario delle due novelle introduttive $^{2}$, che hanno come protagonisti rispettivamente ser Ciappelletto e madonna Oretta. Mentre però l'argomento della Sesta Giornata, proposto da Elissa, la regina di turno, esalta la parola astuta e intelligente («Voglio che domane [...] si ragioni [...] di chi, con alcun leggiadro motto tentato, si riscotesse, o con pronta risposta o avvedimento fuggí perdita o pericolo o scorno»; V Concl. $3^{3}$ ), l'unità tematica della Prima Giornata appare più chiara solamente dopo un attento esame delle singole novelle (Stewart, 1986, p. 33). Tuttavia una spia parziale viene offerta nel preambolo di Fiammetta alla quinta novella: «Sí perché mi piace noi essere entrati a dimostrare con le novelle quanta sia la forza delle belle e pronte risposte [...]» (I 5, 4).

I sopraddetti meccanismi espliciti e impliciti aiutano a capire meglio i rapporti intercorrenti all'interno del macrotesto decameroniano. Dalla sua lettura si evince che esso intende continuare la tradizione della narratio brevis, in particolare - per quanto riguarda soprattutto l'esaltazione della parola come mezzo con cui uno riesce a cambiare/migliorare la realtà - quella del Novellino, dove nel Proemio si afferma

\footnotetext{
${ }^{2}$ Il carattere metaletterario della novella di madonna Oretta è generalmente riconosciuto dalla critica: cf. ad esempio Freedman, 1975-1976, pp. 225-241; Baratto, 1970, pp. 74-76; sul valore metanarrativo non soltanto della novella di Ciappelletto ma addirittura della prima decade del Decameron insiste Picone, 2004b, pp. 57-61, specialmente p. 59.

${ }^{3}$ Tutte le citazioni dal Decameron nel presente articolo sono tratte dall'edizione: Boccaccio, 1992.
} 
l'importanza, nei rapporti privati e pubblici, del «bel parlare», di dare «belli risposi» e, più generalmente, di saper «argomentare e dire e parlare [...] a prode e a piacere» (Novellino, 2008, p. 42) ${ }^{4}$. Perciò è difficile non essere d'accordo con Michelangelo Picone, quando osserva: «Il potere della parola è un'etichetta troppo generica per essere riferita ad una parte soltanto dell'opera; essa è una chiave passepartout che, oltre alla prima e alla sesta giornata, sembra applicarsi a tutto il Centonovelle» (Picone, 2004b, p. 58). Indipendentemente quindi dall'argomento particolare delle singole decadi, annunciato dal re o dalla regina di turno, la forza onnipotente (e a volte prepotente) della parola assume un ruolo significativo nella maggioranza delle novelle, diventando un elemento che accomuna in qualche modo i protagonisti di vari racconti e svolge funzioni non indifferenti nella loro vita. La parola viene da loro impiegata per fargli fuggire i pericoli o perfino la morte, evitare situazioni imbarazzanti, ribaltare la realtà da negativa in positiva, da tragica in «comica» $\mathrm{e}$, in genere, raggiungere un obiettivo desiderato, per lo più l'amore di una donna o di un uomo. L'ingegno che si manifesta nelle pronte risposte, nei «motti leggiadri» o nella capacità fabulatoria dispiegata dai protagonisti che sapientemente confondono il vero con il falso, diventa un'arma potente nella lotta con il destino ostile o con la fortuna «matrigna», oppure uno strumento inestimabile nelle conquiste amorose accompagnate non di rado da inganni che tolgono gli ostacoli rappresentati per lo più da mariti gelosi (e/o sciocchi) o da parenti troppo «affezionati» ai rigidi costumi che impongono alla donna un determinato modus vivendi in famiglia e in società. Insomma, la Fortuna e l'Amore, due forze naturali (l'una esterna, l'altra interna), entrambe poco razionali, che operano nel mondo raccontato nelle novelle, devono ogni volta (o quasi) far fronte ai facta e ai dicta - manifestazioni dell'umana industria e intelligenza.

Siccome l'interesse di Boccaccio è rivolto nel Decameron prevalentemente verso l'ars narrandi e la sua efficacia a livello pratico, perché l'obiettivo che si prefigge è di natura soprattutto estetica (Picone, 2004b, pp. 57-78 passim; Baratto, 1970, pp. 69-89) $)^{5}$, non sorprende il fatto che egli non tenda ad instaurare nel suo capolavoro (o lo faccia raramente) un discorso etico o un insegnamento morale ${ }^{6}$, nonostan-

\footnotetext{
${ }^{4}$ Le due finalità, educativa ed edonistica, esplicitate nell'espressione «a prode e a piacere», vengono riecheggiate nel «diletto [...] e utile consiglio» del Proemio decameroniano $(\S 14)$.

${ }^{5}$ Per quanto riguarda Baratto, nel capitolo «Il diletto narrativo e stilistico» si legge questa osservazione: «Il gusto della novella, che nel Proemio del Decameron trova le sue remote premesse nel ricordo dei piacevoli ragionamenti d'alcuno amico (\$ 4), si inscrive ora in una collettiva consuetudine aristocratica, e si definisce come diletto di favole raccontate da una compagnia esperta, educata all'arte della parola. Svincolato dall'applicazione di schemi cortesi, il Boccaccio propone ora, col Decameron, un testo esemplare per i diletti di una civiltà cittadina» (p. 72). Nel capitolo «Orientamenti morali del Boccaccio», Baratto parla di «un gusto estetico della beffa» e di «una gratuita ammirazione per l'esercizio dell'ingegno» (p. 67).

${ }^{6}$ Sulla qualità della morale presente nel Decameron, nuova rispetto a quella dominante nella letteratura precedente e anche contemporanea al Boccaccio, cf. M. Baratto, 1970, pp. 49-68, dove nella parte
} 
te la rappresentazione dei vizi umani vi occupi uno spazio notevole. I novellatori e le novellatrici raccontano numerosi casi della «commedia umana», a volte con una sottile ironia, a volte con un distacco o compiacimento; il loro commento di fronte alle debolezze umane si scioglie il più delle volte in una sana risata e solo raramente assume la forma di una critica aspra o sprezzante ${ }^{7}$.

Rispetto alla tradizione romanza della narratio brevis, il Decameron, che sta al suo vertice, concede molto spazio alle donne, le destinatarie privilegiate alle quali intende offrire consolazione nelle pene d'amore (per esempio tramite la rappresentazione dei casi in cui la donna riesce a concludere vittoriosamente la vicenda della quale rischiava di rimanere vittima), oltre che distrazione, cosa di cui le donne godono meno degli uomini. Accanto alla complicità dell'autore con le sue lettrici, si osservano altre prove dell'attenzione riservata alle donne, tra cui una netta superiorità numerica della componente femminile della «gentile brigata»e quindi il maggiore spazio concesso alle novellatrici, promotrici importanti della nuova ars narrandi, addirittura ritenute da qualche critico «specchio fedele dell'immagine autoriale» (Picone, 2004b, p. 76). A ciò si potrebbe aggiungere il ruolo attivo che svolgono le donne come protagoniste di molte novelle, brillando spesso più degli uomini (innanzitutto dei loro mariti) per l'intelligenza che si manifesta nell'abilità fabulatoria, nei motti arguti, nonché nella rapidità e astuzia con cui intraprendono azioni rischiose (terminate generalmente con successo), che richiedono molto coraggio o una particolare forza d'animo.

Il Decameron lasciò, com'è risaputo, un'impronta non indifferente sullo sviluppo della commedia cinquecentesca (Borsellino, 1976 e 1989; Stewart, 1986), come fonte inesauribile di trovate, beffe, qui pro quo, invenzioni comiche, grottesche o paradossali, nonché un esemplare repertorio di intrecci e personaggi. Colpisce infatti una forte teatralizzazione di molte novelle, che si manifesta - in alcuni casi con la presenza di «un pubblico» al quale è destinata «la messa in scena», allestita dal personaggio principale, allo stesso tempo «attore»e «regista». Un buon esempio ci è dato dalla celeberrima novella di ser Ciappelletto (I 1) o da quella non meno famosa di frate Cipolla (VI 10), entrambe molto analizzate in questa chiave ${ }^{8}$. Il Boccaccio ha capito più che bene nella sua genialità artistica, che la parola, per mostrare la sua piena efficacia, ha bisogno di essere accompagnata e rafforzata da strategie non verbali, come la gestualità e il linguaggio del corpo, e, naturalmente, quello delle emozioni. In queste «attività», che a volte richiedono vere e proprie abilità

conclusiva si legge: «Si precisa, nel Decameron, una morale di natura intellettuale, se l'intelligenza si pone come un valore decisivo anche nell' ambito della vita quotidiana» (p. 67).

${ }^{7}$ Questo tipo di reazione si fa sentire soprattutto nei confronti dei religiosi indegni di questo nome e lo riscontriamo ad esempio nell'introduzione di Filomena alla terza novella della Terza Giornata che sarà oggetto della nostra analisi.

${ }^{8}$ Per la bibliografia rimando all'edizione da cui provengono le citazioni nel presente articolo: Boccaccio, 1992, pp. LXXXVII-LXXXVIII e CVIII-CIX. 
istrioniche, si dimostrano maestre alcune protagoniste, capaci di gareggiare con ser Ciappelletto e frate Cipolla, ritenuti non solo «i due massimi eroi della menzogna in tutto il Decameron» (Stewart, 1986, p. 31), ma anche i due maggiori esponenti del gioco verbale e teatrale.

Soffermiamoci ora su una di queste protagoniste, finora un po' trascurata dalla critica ${ }^{9}$, il cui ingegno è espressamente lodato da Dioneo nel brevissimo commento che segue la novella III 3, raccontata da Filomena: «Poi che Filomena, finita la sua novella, si tacque, avendo Dioneo con dolci parole molto lo 'ngegno della donna commendato [...]» (III 4, 2). La vena inventiva della donna, le sue doti retoriche e performative, la perfetta riuscita della beffa attuata ai danni di un religioso ${ }^{10}$, della cui credulità e sciocchezza lei si serve come mezzo per raggiungere il suo obiettivo amoroso, meritano più di un approfondimento critico. Si consideri in tal senso soprattutto che, tra la novella di cui lei è protagonista e quella di ser Ciappelletto, si possono indicare alcune somiglianze, a livello non tematico, ma piuttosto strutturale e formale, che riguardano, tra l'altro, lo strumento, l'oggetto e la funzione (nonché funzionalità) della beffa, l'uso della parola e il gioco del contrasto tra realtà e apparenza.

La restrizione dell'argomento della terza serie di novelle rispetto alla seconda (in cui sopra le vicende umane svolge il suo dominio la Fortuna, che alla fine si dimostra benevola), annunciata esplicitamente da Neifile sotto il cui reggimento i novellieri trascorreranno la Terza Giornata, rende il tema della Fortuna ancora più interessante, perché attiva l'intelligenza e la sagacità dei personaggi principali che, grazie alle proprie virtù, tipicamente borghesi (che vanno intese e valutate non nel senso etico ma pratico e - si direbbe - machiavellico avant la lettre; Usher, 2004, p. 100), correggono gli «errori» della Fortuna, non aspettando i suoi «favori»:

Quivi quando noi saremo domenica appresso dormire adunati, avendo noi oggi avuto assai largo spazio da discorrere ragionando, sí perché piú tempo da pensare avrete e sí perché sarà ancora piú bello che un poco si ristringa del novellare la licenzia e che sopra uno de' molti fatti della fortuna si dica, e ho pensato che questo sarà: di chi alcuna cosa molto disiderata con industria acquistasse o la perduta recuperasse. Sopra che ciascun pensi di dire alcuna cosa che alla brigata esser possa utile o almeno dilettevole [...]. (II Concl. 8-9)

\footnotetext{
${ }^{9}$ Per l'analisi di questa novella in chiave comica, cf. M. Baratto, 1970, pp. 281-289; non ne ho trovate altre che abbiano un certo spessore.

${ }^{10} \mathrm{La}$ vittima inconsapevole della beffa della gentildonna fiorentina rimane anche suo marito, che però non appare come personaggio della novella ed è escluso dal gioco teatrale preparato dalla moglie, come se non lo riguardasse minimamente; infatti, la novellatrice (Filomena) ne parla con un distacco, visto che la moglie lo tiene in poco conto e lo tratta in maniera sprezzante, perché lui appartiene alla classe dei laboratores e l'unico suo interesse (almeno agli occhi di lei) è costituto dalla sua attività di tessitore che lo assorbe totalmente.
} 
Il proposito di Boccaccio come autore che - nella confessione fatta nel Proemio rivolto alle donne, destinatarie delle novelle - afferma apertamente di voler rimediare al torto fatto dalla Fortuna al sesso femminile ( $\$ 13)$, si riflette nell' «industria» di tante donne e di vari uomini che si danno da fare per cambiare l'immagine della donna, liberandola dagli schemi imposti dalla rigida morale dell'epoca. La Terza Giornata è particolarmente rappresentativa in questa materia: l'industria illustrata con vari esempi ha il carattere erotico in tutte e dieci le novelle, la maggioranza delle quali (otto) presenta la quête amorosa al di fuori del matrimonio (Usher, 2004, p. 105). In più, tutti gli interventi umani, che operano un rovesciamento della situazione iniziale «voluta» dalla Fortuna, terminano con un «lieto fine».

Dopo queste premesse che mettono a punto gli aspetti importanti per l'argomento indicato nel titolo di questo articolo, passiamo all'esposizione dei momenti cruciali della novella e in seguito all'esame delle tecniche manipolatorie (verbali e emotive) con cui la protagonista, come vedremo, riesce a «incantare» la realtà, rappresentata in particolare da due personaggi maschili. Prima, però, va precisato che si tratta di una delle novelle la cui veridicità è esplicitamente ribadita da Filomena: a suo dire, si tratterebbe di un fatto realmente accaduto di recente a Firenze e, per evitare il possibile sdegno di alcuni che potrebbero ricordarlo, lei dichiara di tacere i nomi di tutte le persone coinvolte nella vicenda (III 3, 5). Non approvando questa potenziale reazione (lo sdegno appunto), aggiunge che l'accaduto dovrebbe piuttosto suscitare il riso, riferendosi forse indirettamente alle parole di Neifile (citate sopra) che raccomandava «il diletto» come finalità imprescindibile delle novelle di questa giornata. Non è fuori luogo ricordare che queste considerazioni rimangono in perfetta sintonia con ciò che in un'altra occasione afferma Fiammetta: «Il partirsi dalla verità delle cose state nel novellare è gran diminuire di diletto negl'intendenti» (IX 5, 5). D'altra parte non c'è dubbio che la veridicità della storia raccontata non vada intesa dai lettori alla stregua di un fatto di cronaca, ma piuttosto di uno verosimile, che potrebbe anche verificarsi in determinate condizioni. In ogni caso la veridicità non è fine a se stessa, ma solo un mezzo per raggiungere la maggior efficacia del racconto (Stewart, 1986, pp. 15-16). Si tratta, insomma, della verità (o veridicità) letteraria, teoricamente formulata già nei trattati di retorica dell'antichità classica (Cicerone, De inv. I, XXI 29; Quintiliano, Ins. orat. IV, II 34).

La protagonista della nostra novella il cui nome, come già sappiamo, non viene svelato, è presentata come «una gentil donna di bellezze ornata e di costumi, d'altezza d'animo e di sottili avvedimenti quanto alcuna altra dalla natura dotata» $(\S 5)$. Se la prima parte della descrizione è un topos ricorrente per tante altre eroine boccacciane, solo poche di esse si distinguono per «l'altezza d'animo» e specialmente per $\mathrm{i}$ «sottili avvedimenti» (segno di una intelligenza fine), un tratto che serve per giustificare i loro exploits, come appunto in questo caso.

L'«errore» della Fortuna - da rimediare - si rivela qui il matrimonio della donna «d'alto legnaggio» $(\S 6)$ con un umile tessitore, che, benché ricco, è tutto 
concentrato sul suo mestiere, trascurando la moglie che lo disprezza e decide di trovarsi un amante, senza però suscitare un minimo sospetto da parte di chiunque. Dopo essersi innamorata di un «assai valoroso uomo e di mezza età» (§ 7), dato che lui non si accorge nemmeno del suo sentimento, escogita un piano assai fine per «conquistarlo», che, curato nei minimi particolari, riuscirà perfettamente. Sfruttando il fatto che il suo amato rimane in ottimi rapporti con un religioso, la donna si reca da quest'ultimo sotto il pretesto di volersi confessare e, finita la confessione vera e propria, si lamenta con il frate di essere perseguitata da quell'uomo che le fa la corte in maniera estremamente fastidiosa e insopportabile per lei, una donna onesta e riservata, che ama suo marito più di se stessa, essendo da lui ricambiata. Abilmente giustifica il fatto di non voler coinvolgere né il marito, né i suoi fratelli per evitare lo scandalo e prega il frate di aiutarla discretamente, distogliendo decisamente il suo amico dall'idea di tenere con lei «questi modi» (§ 13).

Il monaco, avendo creduto a ogni sua parola ( $\$ 14)$, si impegna ad agire in maniera che il suo amico non le dia più fastidio e approfitta della posizione sociale della gentildonna per chiederle un'elemosina, proposta che lei sfrutta per migliorare ancora la propria immagine, mostrandogli l'affezione che nutre per i parenti morti e, «empiutagli nascosamente la man di denari» (§ 16), lo prega di dire le messe per le loro anime.

Intanto «il valente uomo» ( $\$ 17)$, chiamato dal religioso, inizialmente non nasconde lo stupore per i rimproveri e i loro motivi, ma sentite le sue spiegazioni che era stata la donna ad essersi lamentata di lui con il confessore, essendo «piú accorto che 'l santo frate» ( $\$ 20)$, comprende «senza troppo indugio la sagacità della donna» (§ 20) e fingendo di vergognarsi promette di lasciarla in pace; dopodiché, passa incuriosito vicino alla sua casa e dal lieto sguardo della donna può capire di aver ben compreso le sue intenzioni. Nonostante una forte attrazione reciproca, l'uomo non prende nessuna iniziativa; la donna «disiderosa di volerlo piú accendere» (§ 22) si reca di nuovo dal frate e piangendo forte gli racconta un presunto affronto subito dal «valente uomo» che ha osato di mandarle i ricchi doni per raggiungere il suo obiettivo amoroso. Avendoli buttati in grembo al frate, gli chiede sdegnata di restituirli al suo amico non risparmiandogli forti critiche per il suo indegno comportamento. Anche questa visita viene «coronata» da una ricca offerta, consegnata all'avido religioso che neanche ora si accorge di esser stato «uccellato» (§33). Avendo chiamato il presunto corteggiatore, si mostra più adirato di prima e il suo discorso oscilla tra il riecheggiamento delle parole della gentildonna e forti critiche; incontrandosi con deboli proteste da parte dell'uomo, «acceso forte» (§ 35) gli restituisce i presunti doni - prova innegabile delle sue colpevolezze.

Il «valente uomo», lietissimo per la certezza di esser amato dalla donna, non meno che per i doni, passando vicino a casa sua le mostra discretamente la propria gioia e in questa situazione a lei non rimane altro che aspettare il giorno in cui suo marito si deve recare a Genova per il commercio dei panni. Subito dopo la sua par- 
tenza ritorna dal frate e addolorata oltre misura gli svela - nella terza confessione l'inaudita audacia del corteggiatore, il quale, saputo (chissà come?) dell'assenza di suo marito, è stato così insolente da arrampicarsi su un albero da cui poteva facilmente raggiungere la finestra della sua camera da letto e ha cercato di entrarvi; lei però, per fortuna svegliatasi, gliel'ha impedito. Fingendo davanti al frate un dolore infinito, quasi lo accusa della poca efficacia dei suoi precedenti interventi. Questi, dopo aver subito chiamato «il valente uomo», gli dice «la maggior villania che mai a uomo fosse detta, disleale e spergiuro e traditore chiamandolo» (\$ 47). L'altro, inizialmente disorientato, trova il modo di far parlare il frate con più chiarezza e alla fine riesce a calmarlo il meglio che può con le (ennesime) false promesse. La notte seguente sfrutta alla lettera le «istruzioni» ricevute - certo non con questo scopo dal frate e finalmente la donna può appagare il suo desiderio. E «prendendo l'un dell'altro piacere, ragionando e ridendo molto della semplicità di frate bestia» (§ 54) vivono i momenti del sommo diletto.

Il piacere - ovviamente quello estetico e intellettuale - è pure una sensazione che non abbandona la gentile brigata durante e dopo l'ascolto della novella, come si può capire dalle parole rivolte dalla regina (Neifile) a Panfilo a cui tocca parlare, continuare «con alcuna piacevol cosetta» (III 4, 2) il loro «diletto».

Come emerge da questo breve riassunto, la beffa raccontata da Filomena assume una forma insolita ed estremamente raffinata, non riscontrabile in altre novelle, quella che si avvicina ad un minidramma in tre atti, ognuno composto da due scene: nella prima è la donna a dialogare con il frate, nella seconda quest'ultimo ha come interlocutore «il valente uomo» da lui ogni volta chiamato. All'ultimo «atto» segue un epilogo dal quale però viene escluso il personaggio beffato del povero frate, perché l'epilogo non appartiene al mondo finto: le emozioni e i sentimenti dei due amanti, come gioia e desiderio, ma anche il disprezzo manifestato nei confronti del «frate bestia» (con le risate), e indirettamente del marito lontano, tramite la critica degli strumenti adoperati nel suo mestiere: «i lucignoli e' pettini e gli scardassi» (§54), non fanno più parte della «recita».

Il «minidramma» è dall'inizio alla fine inventato e allestito dalla gentildonna che in quanto «regista»e «attrice» in ogni momento controlla la situazione e manipola la realtà con parole e emozioni. La parola da sola non ha, come sappiamo, una sufficiente forza persuasiva, le emozioni che l'accompagnano fanno insorgere altre emozioni che attivano azioni; queste, a loro volta accelerano il raggiungimento dell'obiettivo sperato e quindi il felice (ma non per tutti!) epilogo.

La donna sa che fingendo di essere «altra da sé» può contare su una maggiore fiducia e comprensione da parte del frate. Il finto dolore per la presunta persecuzione amorosa ad opera dell' amico del religioso, definita come uno stato d'assedio («pare che m'abbia posto l'assedio»; $§ 11$ ), fa parte della recita e ben si addice ad una persona casta e onesta (che lei non è); avendo insistito su queste sue presunte qualità, con poche parole delinea il «suo» autoritratto morale, arricchito ulteriormente con 
un gesto di generosità; in realtà però, sfruttando l'inclinazione del monaco all'avidità $^{11}$, con l'offerta del denaro lei lo corrompe facendo accrescere lo zelo con cui egli le dà ascolto. Ma a parte questo, l'inganno diventa possibile soprattutto grazie all'ottusità e l'ingenuità del frate ${ }^{12}$, che avendo a che fare con una donna molto intelligente e audace (entrambe le qualità borghesi fortemente richieste nella realtà municipale fiorentina - e non solo - che devono necessariamente portare al successo, qui limitato alla sfera privata) si lascia facilmente manipolare. Grazie all'uso impeccabile sia delle parole che delle emozioni che le accompagnano, la donna può essere certa che il monaco, date le sue carenze intellettuali (cioè l'incapacità di riflettere in maniera autonoma, ad esempio sull'iniziale stupore del «valente uomo» come reazione alle accuse oggettivamente ingiustificate; $\S 18$ ), si fermerà al senso letterale della performance nella quale è coinvolto senza saperlo e nemmeno senza sospettare che nella «recita» della donna si possa celare un messaggio opposto, destinato al presunto corteggiatore che infatti sarà in grado di recepirlo. Per essere convincente, la donna dispiega una gamma di emozioni che accompagnano «il detto», tra cui sfrutta maggiormente il dolore e la sofferenza, segni dell'offesa subita e dell'impotenza che giustificano sufficientemente il ricorso all'aiuto di un religioso. Il termine «dolore», accanto a quello di «noia» (che qui corrisponde alla molestia o al travaglio) e in alternativa a esso, compare nel primo discorso della donna e viene illustrato dalla sua voglia di piangere: «Io mi dolgo forte, per ciò che questi cosí fatti modi fanno sovente senza colpa alle oneste donne acquistar biasimo [...] ci sono dell'altre donne assai le quali per avventura son disposte a queste cose [...] là dove a me è gravissima noia [...] E detto questo, quasi lagrimar volesse, bassò la testa» (§ 11-13). Il motivo della sofferenza ricompare al termine della confessionelamento, quando lei suggerisce abilmente al frate una cosa fondamentale per la buona riuscita del piano, e cioè di ripetere le sue parole all'amico galante senza nascondergli il dolore da lei provato per il presunto corteggiamento ( «Sicuramente gli dite che io sia stata quella che questo v'abbia detto e siamivene doluta»; § 15), suggerimento che l'ingenuo religioso accoglie alla lettera: «[...] ella medesima, forte di te dolendosi, me l'ha dette» (§ 18).

\footnotetext{
${ }^{11}$ L'avidità dei rappresentanti della Chiesa in Boccaccio, spesso trattata (nei commenti dei novellatori che si leggono nella cornice o all'interno delle novelle da loro raccontate) come una delle debolezze umane che non risparmia lo stato clericale, anzi costituisce quasi un suo vizio «caratterizzante», nel proemio di Filomena a questa novella si riscontra con una critica particolarmente forte (III 3, 3-4). La forte polemica anticlericale della donna che senza mezzi termini attacca (servendosi di uno stile bassissimo) la stoltezza, l'avarizia e l'utilitarismo dei monaci, stona però un po' con la rappresentazione del frate nella novella: è vero che lui pecca di ingenuità, ma molti al suo posto rimarrebbero ingannati, data la superiorità intellettuale e la scaltrezza della donna; è disarmante la sua sciocca semplicità e la sincera convinzione dell'importanza della mediazione chiestagli dalla donna. La sua voglia di approfittare dell'occasione per guadagnare qualcosa viene in parte provocata dalla gentildonna.

${ }^{12}$ L'allusione a entrambe queste «qualità» è presente nell'iniziale caratteristica del frate: «tondo e grosso uomo [...] per ciò che di santissima vita era quasi da tutti avea di valentissimo frate fama» (§ 8).
} 
Si osserva una intensificazione di questa emozione durante le due successive confessioni, man mano che cresce la presunta impudenza del «valente uomo». È significativo che uno spazio sempre maggiore venga dato alla comunicazione non verbale, per cui aumenta l'aspetto teatrale. La scena del secondo «atto» si apre e chiude con il disperato pianto che all'inizio le impedisce di parlare («la donna [...] al santo frate se ne tornò, e postaglisi nella chiesa a sedere a' piedi a piagnere incominciò»; § 22) e alla fine, dopo il discorso pronunciato con un non celato sdegno, accompagna l'esagerato gesto con cui vengono esibite e gettate in grembo al frate le «prove» dell'inaudita insolenza dell'uomo, i doni galanti: «E detto questo, tuttavia piagnendo forte, si trasse di sotto alla guarnacca una bellissima e ricca borsa con una leggiadra e cara cinturetta e gittolle in grembo al frate» (\$ 29). Nella parte centrale del suo discorso viene invece esposta con crescente irritazione la storia del rifiuto dei doni da parte sua, in cui le accuse riferite all'uomo, «sí ardito e sí sfacciato» ( $\$ 26)$, vengono pronunciate con una crescente stizza.

Il culmine della disperazione si ha nell'ultimo «atto», quando la donna arriva turbatissima per raccontare al monaco l'avventura mattutina del «salitor d'alberi»: «Dopo molte querimonie piagnendo gli disse: Padre mio, or vi dich'io bene che io non posso piú sofferire [...] E acciò che voi crediate che io abbia ragione e di piagnere e di ramaricarmi, io vi voglio dire ciò che il vostro amico [...] mi fece stamane poco innanzi matutino» (§ 39). Accanto alla sofferenza, espressa con i mezzi verbali e non verbali, la protagonista finge il sempre maggiore sdegno e l'ira rivolti contro «il diavolo del Ninferno» ( $\$ 39$ ), come chiama l'uomo, ricorrendo a questa eloquente metafora, ambigua per il suo valore assieme tragico e comico data l'ambiguità di tutta la situazione. La temperatura delle sue emozioni sale in una specie di climax ascendente e alla fine esse vengono rivolte anche contro il frate: «Ora ecco disse la donna per questa volta io non vi voglio turbare né disubidire, ma sí adoperate che egli si guardi di piú noiarmi, ché io vi prometto di non tornar piú per questa cagione a voi; e senza piú dire, quasi turbata, dal frate si partí» (\$ 46), senza lasciargli alcuna elemosina.

Un percorso parallelo presenta l'iter emotivo del frate: dapprima, cercando di mantenere la calma, loda l'onestà della donna e le promette di liberarla dall'inopportuno corteggiatore; al secondo incontro, turbato oltre misura ( $\$ 29)$ dopo il concitato discorso della gentildonna, in un primo momento risponde con compassione al suo pianto, poi prova a calmarla e consolarla ed è pronto di «riscaldar gli orecchi» (§ 30) all'uomo che non mantiene le promesse. In seguito, in presenza di quest'ultimo dà libero sfogo a emozioni negative (turbamento, ira, sdegno, rabbia forte) che in ogni successivo incontro con il presunto corteggiatore vengono manifestate in un crescendo che riecheggia la graduale salita delle emozioni «recitate» dalla donna. Se nel primo si limita a un pacato ammonimento e qualche lieve rimprovero («per assai cortese modo il riprese dello intendere e del guardare che egli credeva che esso facesse a quella donna»; § 17), il secondo si svolge in un clima decisamente più teso, perché il frate «di nuovo ingiuriosamente e crucciato parlan- 
dogli, il riprese molto di ciò che detto gli avea la donna che egli doveva aver fatto» (§ 34). In risposta alle tiepide proteste dell'altro, acceso d'ira non esita di chiamarlo «malvagio» (\$ 35). L’ultima scena è molto più colorita, in perfetta corrispondenza con la movimentata performance della donna: il turbamento del frate raggiunge il culmine già nel colloquio con la donna («fu il piú turbato uomo del mondo»; § 42), il che si traduce nel modus loquendi per metafore che potrebbero concorrere con il linguaggio dantesco nelle Malebolge: «lasci fare a me, a veder se io posso raffrenare questo diavolo scatenato, che io credeva che fosse un santo. E se io posso tanto fare che io il tolga da questa bestialità, bene sta» (\$ 45). Su questo tono il frate continua la predica furibonda rivolta allo «spergiuro» e «svergognato» amatore delle mogli altrui, nella quale moltiplica gli epiteti «caratterizzanti» lui e il suo vizio; incollerito dalla poca memoria dell'altro (che ovviamente non sa cosa ha fatto questa volta e stuzzica il religioso che glielo dica), adopera una ironia che sa di comico: $« \mathrm{Hi}$, meccere: ${ }^{13}$ ecco onesto uomo! è divenuto andator di notte, apritor di giardini e salitor d'alberi!» (\$ 50).

Se il frate è ignaro di partecipare a un dramma finto e perciò «recita» senza saperlo la parte impostagli dalla gentildonna, ciò non si può dire del «valente uomo» che invece «recita» passivamente, assecondando il gioco, attento allo sviluppo della situazione e ai messaggi che riesce a recepire dai discorsi del frate, il quale riecheggiando le parole della gentildonna, in realtà destinate all'altro, le arricchisce, come abbiamo visto, con alcune concitate critiche.

Il comportamento dell'uomo, divenuto sempre più irritante per il frate, rimane in funzione della riuscita della beffa. Le sue risposte alla critica del monaco, che si fa sempre più aspra, sono finalizzate a non destare il sospetto di chi lo riprende, e, d'altra parte, a rassicurare la donna, accertandola della sua piena complicità.

Rispetto ad altri due personaggi, il «valente uomo» rimane necessariamente «sbiadito» come carattere di questo dramma comico. Privo di una vera e propria personalità, egli si lascia «plasmare» dalla gentildonna. Ma la sua passività si riesce a spiegare anche diversamente. Nel suo caso non si tratta di uno di quegli amanti infuocati e impazienti - non pochi nel Decameron - che, avendo perso il modo razionale di pensare e agire commettono vari errori e devono la «salvezza» spesso al sangue freddo e alla sagacità delle loro donne. All'inizio non si accorge nemmeno di essere oggetto del desiderio erotico, perciò reagisce - ignaro - alle prime accuse con stupore, ma dal momento in cui (ancora nel corso del primo colloquio con il frate) coglie le intenzioni della gentildonna, agisce di conseguenza, accettando le accuse, difendendosi con poca convinzione e mostrando come unica emozione la (non troppo accentuata) vergogna. Il «valente uomo» è un calcolatore che coglie la fortuna al volo, senza alcun ritegno morale o altro, una cosa che gli viene tanto più facile quanto più il fantomatico marito è tagliato fuori non solo dalla vicenda, ma quasi dalla vita della donna.

${ }^{13}$ V. Branca osserva nella nota che il frate usando la parola «meccere» invece di «messere» imita il modo di parlare dei bambini (Boccaccio, 1992, p. 358, n. 1). 
È lei che dall'inizio alla fine tira le fila della commedia, grazie al suo ingegnoso piano e specialmente alle strategie verbali e non verbali con cui lo attua. La storia da lei inventata con parole ed emozioni, richiede dai due uomini un determinato comportamento, da lei in qualche modo previsto, per cui ciò che in un primo momento si presenta come illusorio, alla fine diventa vero. Si può dire che la protagonista di questa novella è una incantatrice della realtà. E in questo almeno supera ser Ciappelletto, il manipolatore più clamoroso in tutto il Decameron. Tra lui e lei, nonché tra le loro rispettive storie, esistono alcuni punti di contatto: l'uno e l'altra si distinguono per un'intelligenza fine e audace, priva di scrupoli (anche se molto di più nel caso di ser Ciappelletto, l'uomo più malvagio del mondo); come oggetto della beffa e il suo strumento entrambi scelgono un frate «santo», ma estremamente ingenuo; per realizzarla sfruttano la confessione, divenuta la struttura portante del gioco teatrale, stravolgendo il suo senso (e rendendola blasfema, speciamente ser Ciappelletto) tramite il racconto delle menzogne che permettono a loro di creare una falsa immagine di se stessi, l'opposto ironico di quella vera, che rimane in funzione dell'obiettivo che si propongono. La beffa in entrambi i casi rovescia la situazione iniziale, ma non è fine a se stessa, in quanto serve a raggiungere qualcosa d'altro.

Le affinità riguardano pure i rispettivi frati, santi, ingenui e sciocchi, che proprio grazie a queste «doti» possono diventare oggetto e strumento della beffa. Ma diventano molto di più, perché senza volerlo né saperlo servono una causa assolutamente inconciliabile con la posizione e il ruolo che hanno nella società: l'uno diventa sacrilego, l'altro ruffiano.

Se queste sono le maggiori somiglianze, non mancano pure le differenze. Anzittutto la confessione blasfema di Ciappelletto ha dei risvolti pubblici, che si estendono a tutta la comunità. Essi mancano del tutto nell'altra storia, rigorosamente privata. Ma ciò che consente di dare, per così dire, il primato alla protagonista della novella di Filomena, è la capacità di «incantare» la realtà. Ciappelletto riesce più di lei a stravolgere la realtà (lui, l'uomo più scellerato che esista, sarà venerato dopo la morte come un santo), ma gli effetti della sua beffa vanno al di là delle sue previsioni e intenzioni. Se prendiamo in considerazione un notevole aumento della devozione religiosa della gente grazie alla sua confessione e ciò che ne risulta, possiamo constatare che la situazione gli è sfuggita di mano, il che non si può mai dire della gentildonna. Alla fine lo scherzo che ser Ciappelletto credeva di tirare al destino gli si rivolge contro, perché a livello - per così dire - metafisico, da beffatore diventa beffato.

Pure l'«incanto» della gentildonna fiorentina si rivela più potente e «pericoloso» di quanto uno possa pensare, ma in un senso completamente diverso: esso oltrepassa infatti i confini narrativi non solo della novella, ma pure quelli della cornice, dal momento che Filomena sospira a Dio che nella sua misericordia consenta a lei e a «tutte l'anime cristiane che voglia n'hanno» (§ 55) di sperimentare la gioia (delle notti trascorse tra le braccia di un amante) pari a quella della sua protagonista. 


\section{BIBLIOGRAFIA}

Baratto, M. (1970). Realtà e stile nel «Decameron». Vicenza: Neri Pozza.

Boccaccio, G. (1992). Decameron (V. Branca, Ed.). Torino: Einaudi.

Borsellino, N. (1976). Rozzi e intronati. Esperienze e forme di teatro dal «Decameron»al «andelaio». Roma: Bulzoni.

Borsellino, N. (1989). La tradizione del comico. L'eros, l'osceno, la beffa nella letteratura italiana da Dante a Belli. Milano: Garzanti.

Freedman, A. (1975-1976). Il cavallo del Boccaccio: fonte, struttura e funzione della metanovella di madonna Oretta. Studi sul Boccaccio, IX, 225-241.

Mouchet, V. (Ed.) (2008). Il Novellino. Milano: BUR.

Picone, M. (1993). Il «Decameron». In F. Brioschi, C. Di Girolamo (Eds.), Manuale di letteratura italiana. Storia per generi e problemi, I: Dalle origini alla fine del Quattrocento (pp. 625-654). Torino: Bollati Boringhieri.

Picone, M. (2004a). Il «Decameron» come macrotesto: il problema della cornice. In M. Picone, M. Mesirca (Eds.), Lectura Boccaccii Turicensis. Introduzione al «Decameron» (pp. 9-31). Firenze: Cesati.

Picone, M. (2004b). Il principio del novellare: la Prima Giornata. In M. Picone, M. Mesirca (Eds.), Lectura Boccaccii Turicensis. Introduzione al «Decameron» (pp. 57-78). Firenze: Cesati.

Stewart, P. D. (1986). La novella di madonna Oretta e le due parti del «Decameron». In P. D. Stewart, Retorica e mimica nel «Decameron» e nella commedia del Cinquecento (pp. 19-38). Firenze: Olschki.

Usher, J. (2004). Industria e acquisto erotico: la Terza Giornata. In M. Picone, M. Mesirca (Eds.), Lectura Boccaccii Turicensis. Introduzione al «Decameron» (pp. 99-114). Firenze: Cesati. 